\title{
Gestión de riesgos en el sistema de distribución de gas natural de Lima y Callao según lineamientos "Recommendations on transmission and distribution practice" y la gestión de los riesgos del proyecto del PMI (2008) \\ Roger Orlando Luján Ruiz*
}

Recibido: 10/03/13 Aceptado: 25/03/14

\section{RESUMEN}

El presente estudio trata sobre el análisis de riesgos en el sistema de distribución de gas natural en Lima y Callao, usando una matriz de riesgos que ha permitido calificar los niveles de riesgo, de acuerdo a los lineamientos de la recomendación práctica sobre distribución de gas "Recommendations on Transmission and Distribution Practice", publicado por The Institution of Gas Engineers de Gran Bretaña y la Gestión de los Riesgos del Proyecto del PMI(2008).

Este estudio se realizó en el ducto troncal del sistema operado por Cálidda-Gas Natural del Perú, desde el ingreso de gas (City Gate) ubicado en Lurín, hasta la Estación Terminal Callao.

Palabras clave: distribución de gas natural gestión de riesgos, medidas de mitigación

Risk MANAGEMENT SYSTEM IN THE NATURAL gas distribution of Lima and Callao ACCORDING GUIDELINES "RECOMMENDATIONS ON TRANSMISSION AND DISTRIBUTION PRACTICE" and Risk Management of the PMI Project (2008)

\section{ABSTRACT}

The purpose of this research study is to analyze quantitative operational risk according to the guidelines of the practice recommendation "Recommendations on Transmission and Distribution Practice", published by The Institution of Gas Engineers of Great Britain and the PMBOK . Chapter 11 , Managing Project Risk

This study was conducted in the Trunk pipeline system operated by Cálidda the Peru - Natural Gas from the City Gate located in Lurín , to Callao Terminal Station

The purpose of this study was to determine the levels of individual risk and safety of the general public due to the operation of the pipeline that operates Cálidda Trunk. In cases where the individual or social risks are considered intolerable compared to international standards mitigation measures associated with the operation to levels that can be considered consistent with best industry practices recommended risk.

Keywords: mitigation measures, management risk natural gas distribution

\section{INTRODUCCIÓN}

La seguridad de las personas o sus propiedades es una de las metas primordiales de las instalaciones industriales donde existe la posibilidad que un accidente cause explosiones, incendios o derrames de sustancias tóxicas.

El desarrollo de un país está ligado a un adecuado aprovisionamiento y acceso oportuno a los diversos tipos de energía existentes, pues la disponibilidad de ésta juega un rol fundamental en la vida y economía de un país. Es prácticamente imposible el desarrollo de un país si no dispone de fuentes de energía seguras y a costos razonables, en línea con lo cual, los hidrocarburos (gas natural y petróleo) constituyen la primera fuente de energía del mundo. (Chambergo, 2009, p. 21)

En las instalaciones industriales se encuentran los ductos enterrados de transporte de energía (combustibles líquidos o gaseosos).

Una de las formas más eficientes para definir las medidas que son necesarias para evaluar la seguridad de las personas y sus propiedades es el análisis de riesgo.

Para fines del presente trabajo, "riesgo" se define como la medida de la probabilidad y magnitud de un evento perjudicial que es válida sólo para un periodo de tiempo especificado. El análisis de riesgo es un proceso sistemático de identificación y análisis cuantitativo y/o cualitativo de la probabilidad y magnitud asociados a un sistema.

\section{MARCO TEÓRICO}

El gas y el petróleo representan más de la mitad del consumo de energía primaria de la humanidad. Uno de los factores que hace que el petróleo sea tan valioso es su extrema versatilidad, como fuente de energía y como materia prima. La facilidad de transporte y almacenamiento permite usarlo como fuente de energía prácticamente en cualquier lugar.

El crudo de petróleo está compuesto por alrededor de un millón de diferentes tipos de moléculas, el gas natural está compuesto tan solo de algunas. (Raymond, Leffler, 2006, p. 103)

El petróleo y el gas tienen su principal origen en la descomposición bacterial de residuos de plantas y materia orgánica depositada

\footnotetext{
* IIngeniero Químico - Universidad Mayor de San Marcos. E-mail: rogger159@yahoo.com
} 
en lagos y mares, con otros sedimentos a través del tiempo desde hace millones de años. Estos sedimentos abarcan granos de material erosionado de las rocas, los cuales se degradaron $y$ se petrificaron constituyéndose en rocas sedimentarias.

El gas natural es la mezcla de hidrocarburos ligeros, fundamentalmente metano (90-99 por $100 \mathrm{ppm}$ ) y pequeñas cantidades de otros (etano, propano) e impurezas y sustancias indeseables tales como: agua, nitrógeno, dióxido de carbono, ácido sulfhídrico gaseoso y helio. Estos se eliminan antes de que el gas llegue al usuario final. (Parra, 2003, p. 46).

La transformación de la materia orgánica en petróleo y gas ocurre gradualmente bajo la tierra por incremento de la presión, temperatura y ausencia de aire. También se considera que después de formado el petróleo y el gas, migraron lentamente de los poros de las rocas sedimentarias, bajo la influencia de las fuerzas naturales, especialmente la gravedad. Esta migración era interrumpida al encontrarse con formaciones no porosas y de baja permeabilidad, debido a la compactación o cambio en los estratos que constituyen las llamadas trampas estratigráficas de petróleo y/ o gas.

En los pozos de producción de petróleo hay grandes cantidades de gas que al extraer el crudo a la superficie es arrastrado, para luego ser separado de éste en los separadores de las estaciones de flujo. En este proceso de separación, el crudo es el gas natural enviado a los sistemas de almacenamiento o a las refinerías donde es procesado; mientras que el gas rico que se obtiene, que en este punto es una mezcla de hidrocarburo de forma líquida y gaseosa, comienza su tratamiento y proceso de separación mediante el traslado a las plantas de procesamiento y acondicionamiento de gas y luego al sistema de transmisión y distribución.

La expansión del mercado del gas natural, en países de territorios grandes, es afectada por la falta de la infraestructura para su transporte. La necesidad de incentivar el uso masificado de gas natural, fuera de los grandes centros de consumo, busca disponer al mercado un combustible limpio, eficiente, barato, abundante y que cumpla con los parámetros de emisiones fijados por los órganos de medio ambiente, además de estimular el uso del gas natural como fuente de energía para uso industrial, doméstico y automotriz. El uso del gas natural tiene fuerte impacto sobre los costos industriales, logísticos y ambientales en el transcurso de la sustitución del diesel, gasolina (Lopez, 2010, p. 12).
Podemos decir que los hidrocarburos, como objetos de estudio de los científicos sociales, han sido abordados desde varias perspectivas, así, algunas aproximaciones al tema han sido de tipo histórico-político, otras han hecho énfasis en los aspectos económicos, también se encuentran aquellas interpretaciones respecto a las formas jurídicas de los contratos, o las referidas a temáticas medioambientales ante la contaminación producida por la industria petrolera. A pesar de esta variedad de enfoques, existe un tema que de manera recurrente es considerado en casi todas las investigaciones: la inevitable relación de poder que se establece entre estados y empresas, entre compañías petroleras y grupos sociales de toda índole, o todos ellos a la vez, en torno a la explotación de los hidrocarburos. (Fernández, 2009, p. 57)

\subsection{Teoría de los riesgos}

Según el PMI (2008) la gestión de los riesgos del proyecto está conformada por seis actividades:

- Planificación

- Identificación

- Análisis cualitativo

- Análisis cuantitativo

- Planificación de la respuesta

- Monitoreo y control de riesgos

Herramientas y Técnicas del Análisis Cuantitativo de los Riesgos

- Técnicas de recopilaciones y representación de datos:

\section{Entrevistas}

Distribuciones de probabilidades

- Técnicas de análisis cuantitativo de riesgos y de modelado:

- Análisis de sensibilidad

- Análisis del valor monetario esperado

- Análisis mediante el árbol de decisiones

- Modelado y simulación

- Juicio de expertos:

- Es requerido para identificar impactos potenciales de los costos y los plazos".

Herramientas y Técnicas del Monitoreo y Control de Riesgos

- "Reevaluación de los riesgos

- Auditoria de los riesgos 
- Análisis de variación y de tendencias

- Medición del rendimiento técnico.

- Análisis de reserva.

- Reuniones sobre el estado de situación".

\section{Evaluación del riesgo}

Evaluar sobre una base recurrente la probabilidad e impacto de los riesgos identificados, usando métodos cualitativos y cuantitativos. La probabilidad e impacto de los riesgos inherentes y residuales deberían ser determinados individualmente, por categoría, o sobre la base de un portafolio.

\section{Respuesta a los riesgos}

Identificar al propietario de los riesgos y a los propietarios de los procesos afectados, y desarrollar y mantener una respuesta a los riesgos para asegurar que el costo efectividad de los controles y medidas de seguridad permita mitigar la exposición a los riesgos en una base permanente. La respuesta a los riesgos debería identificar las estrategias de riesgos tales como evitar, reducir, compartir o aceptar. En el desarrollo de la respuesta, considerar los costos y los beneficios y seleccionar las respuestas que restringen los riesgos residuales dentro de los niveles definidos de la tolerancia de los riesgos.

\section{Mantener y Monitorear el Plan de Acción contra los Riesgos}

Priorizar y planear el control de las actividades en todos los niveles para implementar las respuestas a los riesgos identificados como necesarios, incluyendo la identificación de los costos, beneficios, y responsabilidades para la ejecución. Buscar la aprobación para las acciones recomendadas y aceptación de cualquier riesgo residual, y asegurar que las acciones comprometidas sean asignadas a los propietarios de los procesos. La ejecución del monitoreo de los planes, y reportes de cualquier desviación a la Alta Dirección.

\section{METODOLOGÍA DE LA INVESTIGACIÓN}

Los componentes principales de esta investigación son del tipo documental resultando esencial conocer todos los aspectos, conceptos y términos que se encuentran relacionado con el tema, permitiendo presentar en un contexto la información requerida en el trabajo de diseño de ingeniería de detalle, de acuerdo a un orden de relevancia establecido con respecto al tema de trabajo.
En atención al diseño aplicable para esta investigación, es de campo, ya que se realizó un análisis sistemático de problemas de la realidad, con el propósito bien sea de describirlos, explicar sus causas y efectos, o predecir su ocurrencia. Los datos de interés fueron recogidos en forma directa de la realidad, en este sentido se trata de investigaciones a partir de datos originales 0 primarios.

El esquema del diseño de la investigación es como sigue:

a. Entrevista al personal dentro de su área de trabajo sobre los peligros y riesgos asociados en sistema de distribución de gas natural.

b. Identificación de riesgos y sus medidas de control existentes

c. Evaluación de riesgos a través de puntajes asignados a la probabilidad del riesgo y sus consecuencias en el personal y el patrimonio de la empresa

d. Establecimientos de medidas de control.

\section{Ámbito de aplicación}

El Sistema de Distribución de Gas Natural en Lima y Callao, se circunscribe en la empresa Cálidda - Gas Natural de Lima y Callao S.A,, objeto del estudio, sus actividades principales son: diseñar, construir y operar el sistema de distribución de gas natural en el departamento de Lima y Callao.

\section{Técnicas de análisis}

- Marco normativo del Gas Natural. Dentro del plan de mejoramiento continuo de gas Natural, se cuenta con un proceso de normalización, el cual tiene por objeto reglamentar y controlar todos los procesos vitales para el correcto funcionamiento del sistema y de esta manera, ofrecer un alto grado de calidad y seguridad para sus clientes según Lineamientos "Recommendations on Transmission and Distribution Practice" y la Gestión de los Riesgos del Proyecto del PMI (2008).

- Matriz de identificación y evaluación cualitativa de riesgos en la distribución de gas natural según PMI (2008)

- "Modelo de un Plan de contingencia para emergencias para la distribución de gas natural para Lima y Callao" 
- Matriz de Identificación de Peligros, Valoración y Control de Riesgos en la distribución de gas natural de Lima y Callao

- El análisis de riesgos permite prever y corregir los accidentes y sus consecuencias en las plantas industriales.

\section{Diagnóstico de gestión de riesgos}

El análisis de riesgos incluye un formato guía para la identificación de riesgos en el sistema de distribución de gas natural, mostrado en el cuadro $\mathrm{N}^{\circ} 4$. Los riesgos significativos corresponden a los riesgos con calificación alta y moderada.

Cuando el riesgo es moderado o mayor está asociado con consecuencias extremadamente dañinas, se precisará una acción posterior para establecer, con más precisión, la probabilidad de daño como base para determinar la necesidad de mejora de las medidas de control.

\section{Cuadro 1.}

\section{MATRIZ DE RIESGOS}

\begin{tabular}{|c|c|c|c|c|c|}
\hline \multicolumn{6}{|c|}{ CUADERNILLO DE TRABAJO PARA LA EVALUACION DE RIESGOS } \\
\hline & \multicolumn{5}{|c|}{ Consecuencia } \\
\hline & \multirow{2}{*}{$\begin{array}{c}1 \\
\text { Insigni ficante }\end{array}$} & \multirow{2}{*}{$\begin{array}{c}2 \\
\text { Menor } \\
\end{array}$} & \multirow{2}{*}{$\begin{array}{c}3 \\
\text { Moderada }\end{array}$} & \multirow{2}{*}{$\begin{array}{c}4 \\
\text { Mayor }\end{array}$} & \multirow{2}{*}{$\begin{array}{c}5 \\
\text { Catastrofica }\end{array}$} \\
\hline Probabilidad & & & & & \\
\hline Siempre (5) & Alto & Alto & Extremo & Extremo & Extremo \\
\hline $\begin{array}{l}\text { Muy Probablemente } \\
\text { (4) }\end{array}$ & Moderado & Alto & Alto & Extremo & Extremo \\
\hline Probablemente (3) & Bajo & Moderado & Alto & Extremo & Extremo \\
\hline Poco Probable (2) & Bajo & Bajo & Moderado & Alto & Extremo \\
\hline & Bajo & Bajo & Moderado & Moderado & Alto \\
\hline
\end{tabular}

\begin{tabular}{|c|c|c|c|c|c|}
\hline \multicolumn{6}{|c|}{ CUADERNILLO DE TRABAJO PARA LA EVALUACION DE RIESGOS } \\
\hline & \multicolumn{5}{|c|}{$\begin{array}{l}\text { Consecuencia } \\
\end{array}$} \\
\hline & \multirow{2}{*}{$\begin{array}{c}1 \\
\text { Insigni ficante }\end{array}$} & \multirow{2}{*}{$\begin{array}{c}2 \\
\text { Menor }\end{array}$} & \multirow{2}{*}{$\begin{array}{c}3 \\
\text { Moderada }\end{array}$} & \multirow{2}{*}{$\begin{array}{c}4 \\
\text { Mayor }\end{array}$} & \multirow{2}{*}{$\begin{array}{c}5 \\
\text { Calastrofica }\end{array}$} \\
\hline Probabilidad & & & & & \\
\hline Siempre (5) & 11 & 16 & 20 & 23 & 25 \\
\hline $\begin{array}{l}\text { Muy Probablemente } \\
\text { (4) }\end{array}$ & 7 & 12 & 17 & 21 & 24 \\
\hline Probablemente (3) & 4 & 8 & 13 & 18 & 22 \\
\hline Poco Probable (2) & 2 & 5 & 9 & 14 & 19 \\
\hline Rara $v e z(1)$ & 1 & 3 & 6 & 10 & 15 \\
\hline
\end{tabular}

Fuente: Elaboración propia. 


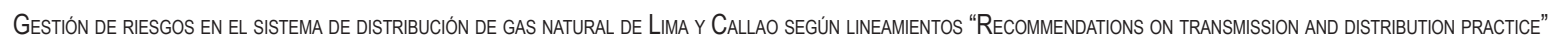
Y LA GESTIÓN DE LOS RIESGOS DEL PROYECTO DEL PMI (2008)

\begin{tabular}{|c|c|c|c|c|}
\hline \multicolumn{5}{|c|}{ Consecuencias } \\
\hline Nivel & Seguridad & Salud & Medio Ambiente & Económicas \\
\hline A & $\begin{array}{l}\text { Sin efectos/ ningún } \\
\text { herido. }\end{array}$ & Sin efectos. & Sin efectos. & \\
\hline B & $\begin{array}{l}\text { Heridos leves/ } \\
\text { Tratamiento } \\
\text { médico. }\end{array}$ & $\begin{array}{l}\text { Posibilidad de problemas } \\
\text { de salud temporales. }\end{array}$ & $\begin{array}{l}\text { Ligero daño dentro de una } \\
\text { zona controlada. }\end{array}$ & $10-50 \mathrm{~K} \$$ \\
\hline C & $\begin{array}{l}\text { Lesiones leves con } \\
\text { hospitalización. }\end{array}$ & $\begin{array}{l}\text { Posibilidad de problemas } \\
\text { de salud permanentes. }\end{array}$ & $\begin{array}{l}\text { Ligero daño, con una } \\
\text { infracción o denuncia. }\end{array}$ & $50-100 \mathrm{~K} \$$ \\
\hline D & $\begin{array}{l}\text { Lesiones } \\
\text { importantes con } \\
\text { daños irreversibles. }\end{array}$ & $\begin{array}{l}\text { Mucha probabilidad } \\
\text { de problemas de salud } \\
\text { permanentes. }\end{array}$ & $\begin{array}{l}\text { Efectos significativos con } \\
\text { repetidas infracciones y } \\
\text { muchas denuncias. }\end{array}$ & $100-250 \mathrm{~K} \$$ \\
\hline$E$ & $\begin{array}{l}\text { Discapacidad total } \\
\text { permanente /De } 1 \text { a } \\
3 \text { víctimas mortales. }\end{array}$ & $\begin{array}{l}\text { Mucha probabilidad } \\
\text { de problemas de salud } \\
\text { permanentes con alguna } \\
\text { víctima mortal. }\end{array}$ & $\begin{array}{l}\text { Efectos importantes con } \\
\text { infracciones prolongadas y } \\
\text { daños generalizados. }\end{array}$ & $250-300 \mathrm{~K} \$$ \\
\hline $\mathbf{F}$ & $\begin{array}{l}\text { Múltiples víctimas } \\
\text { mortales. }\end{array}$ & $\begin{array}{l}\text { Mucha probabilidad } \\
\text { de problemas de salud } \\
\text { permanentes con múltiples } \\
\text { víctimas mortales. }\end{array}$ & $\begin{array}{l}\text { Efectos masivos con daños } \\
\text { severos persistentes. }\end{array}$ & $>500 \mathrm{~K} \$$ \\
\hline
\end{tabular}

Fuente: Elaboración propia.

\section{Cuadro 2.}

\begin{tabular}{|c|c|c|c|c|c|c|c|}
\hline \multirow{8}{*}{ 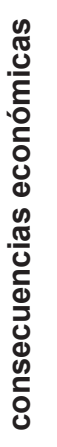 } & \multicolumn{7}{|c|}{ Máximo consecuencias de Seguridad/Salud/Medio Ambiente } \\
\hline & & A & B & C & D & $\mathbf{E}$ & $\mathbf{F}$ \\
\hline & A & 0 & 10 & 45 & 75 & 90 & 100 \\
\hline & B & 10 & 25 & 50 & 80 & 90 & 100 \\
\hline & C & 20 & 35 & 70 & 85 & 95 & 100 \\
\hline & D & 50 & 65 & 75 & 90 & 98 & 100 \\
\hline & $E$ & 75 & 85 & 90 & 95 & 100 & 100 \\
\hline & $\mathbf{F}$ & 85 & 90 & 100 & 100 & 100 & 100 \\
\hline
\end{tabular}

\begin{tabular}{|c|c|}
\hline $0-24$ & A \\
\hline $25-49$ & B \\
\hline $50-69$ & C \\
\hline $70-84$ & D \\
\hline $85-94$ & E \\
\hline $95-100$ & $F$ \\
\hline
\end{tabular}

Fuente: Elaboración propia. 
Cuadro 3. Niveles de consecuencias globales.

\begin{tabular}{|c|c|c|}
\hline Nivel & Definición & MTBF (años) y f \\
\hline Muy alta & Ocurre varias veces al año en esta planta. & $\begin{aligned} f & \geq 0.1 \\
\text { MTBF } & <1 \text { año }\end{aligned}$ \\
\hline Alta & Ocurre al menos una vez cada tres años en esta planta. & $\begin{array}{l}0.015 \leq \mathrm{f}<0.1 \\
1 \leq \mathrm{MTBF} \leq 3\end{array}$ \\
\hline Moderada & Ha ocurrido alguna vez en esta planta. & $\begin{array}{l}0.01 \leq f<0.015 \\
3<M T B F \leq 10\end{array}$ \\
\hline Baja & $\begin{array}{l}\text { No ha ocurrido nunca en esta planta, pero es probable que } \\
\text { ocurra según la experiencia en esta industria. }\end{array}$ & $\begin{array}{l}0.005 \leq \mathrm{f}<0.01 \\
10<\mathrm{MTBF} \leq 28\end{array}$ \\
\hline Muy baja & $\begin{array}{c}\text { No ha ocurrido nunca en esta planta, y es improbable que } \\
\text { ocurra según la experiencia en esta industria. }\end{array}$ & $\begin{array}{l}f<0.0005 \\
\text { MTBF }>28\end{array}$ \\
\hline
\end{tabular}

Fuente: Elaboración propia.

\section{CUANTIFICACIÓN DEL RIESGO}

Para dar un valor numérico a las consecuencias, utilizamos los valores numéricos obtenidos de la Tabla 2 (donde 1 representa las mínimas consecuencias y 100 las máximas) y los elevamos al cuadrado expandiéndolos a un rango de 0-1000.

La razón de elevar al cuadrado los valores, es que las consecuencias de las ocurrencias no se distribuyen de forma lineal. Es decir, una evolución de 25 a 35 en el nivel de consecuencias, no debe tener la misma importancia que pasar 85 a 95. De esta forma, elevando al cuadrado los niveles, obtendríamos una evolución de 625 a 1225 (una diferencia de 600) frente a una de 7225 a 9025 (una diferencia de 1800).

Este proceso no tiene influencia en el ranking de riesgos que obtendremos pero sí, modifica la contribución que cada modo de fallo aporta al riesgo total de la planta.

El riesgo para cada modo de fallo lo evaluamos de la siguiente manera:

Riesgo $=$ Consecuencia $* 1 /$ (MTBF (años) $\left.{ }^{*} 365\right)$

De tal forma que las escalas podrían quedar de la siguiente manera:

Matriz de riesgo

\begin{tabular}{|c|c|c|c|c|c|c|c|}
\hline & & \multicolumn{6}{|c|}{ Consecuencias } \\
\hline \multirow{6}{*}{ 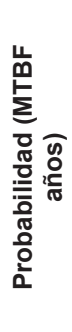 } & & $0-576$ & $577-2400$ & $2401-4761$ & $4762-7056$ & $7057-8836$ & $8837-10 K$ \\
\hline & MTBF $<1$ & S & $S$ & $\mathrm{H}$ & $\mathrm{H}$ & $\mathrm{H}$ & $\mathrm{H}$ \\
\hline & $1 \leq \mathrm{MTBF} \leq 3$ & M & $S$ & $S$ & $\mathrm{H}$ & $\mathrm{H}$ & $\mathrm{H}$ \\
\hline & $3<\mathrm{MTBF} \leq 10$ & M & M & $S$ & $S$ & $\mathrm{H}$ & $\mathrm{H}$ \\
\hline & $10<M T B F \leq 28$ & L & M & M & $S$ & $S$ & $\mathrm{H}$ \\
\hline & MTBF>28 & $\mathrm{L}$ & L & M & $\mathrm{M}$ & S & S \\
\hline
\end{tabular}

Fuente: Elaboración propia. 
Cuadro 4. Análisis de riesgos.

\begin{tabular}{|c|c|c|c|c|c|}
\hline \multicolumn{5}{|c|}{ CONTROL DE VERSIONES } \\
\hline Versión & Hecha por & Revisada por & Aprobada por & Fecha & Motivo \\
\hline 01 & R. Lujan & & & $10 / 08 / 13$ & VERSIÓN ORIGINAL \\
\hline
\end{tabular}

\begin{tabular}{|c|c|}
\hline NOMBRE DEL PROYECTO & SIGLAS DEL PROYECTO \\
\hline $\begin{array}{c}\text { DISTRIBUCIÓN DE GAS NATURAL PARA CLIENTES } \\
\text { RESIDENCIALES Y COMERCIALES }\end{array}$ & LUJÁN \\
\hline
\end{tabular}

\begin{tabular}{|c|c|c|c|c|c|}
\hline Probabilidad & $\begin{array}{c}\text { Valor } \\
\text { Numérico }\end{array}$ & Impacto & $\begin{array}{c}\text { Valor } \\
\text { Numérico }\end{array}$ & Tipo de Riesgo & Probabilidad x Impacto \\
\hline Muy Improbable & 0.1 & Muy Bajo & 0.05 & Muy Alto & mayor a 0.50 \\
\hline $\begin{array}{c}\text { Relativamente } \\
\text { Probable }\end{array}$ & 0.3 & Bajo & 0.10 & Alto & menor a 0.50 \\
\hline Probable & 0.5 & Moderado & 0.20 & Moderado & menor a 0.30 \\
\hline Muy Probable & 0.7 & Alto & 0.40 & Bajo & menor a 0.10 \\
\hline Casi Certeza & 0.9 & Muy Alto & 0.80 & Muy Bajo & menor a 0.05 \\
\hline
\end{tabular}

\begin{tabular}{|c|c|c|c|c|c|c|c|c|c|}
\hline $\begin{array}{l}\text { Código } \\
\text { del } \\
\text { Riesgo }\end{array}$ & $\begin{array}{c}\text { Descripción del } \\
\text { Riesgo }\end{array}$ & Causa Raíz & Trigger & $\begin{array}{l}\text { Entregables } \\
\text { Afectados }\end{array}$ & $\begin{array}{c}\text { Estimación } \\
\text { de proba- } \\
\text { bilidad }\end{array}$ & $\begin{array}{l}\text { Objetivo } \\
\text { Afectado }\end{array}$ & $\begin{array}{l}\text { Estima- } \\
\text { ción de } \\
\text { impacto }\end{array}$ & $\begin{array}{c}\text { Prob } x \\
\text { Impacto }\end{array}$ & $\begin{array}{l}\text { Tipo de } \\
\text { Riesgo }\end{array}$ \\
\hline \multirow{5}{*}{ R001 } & \multirow{5}{*}{\begin{tabular}{|l|} 
Paralización \\
de obra por \\
personal de \\
sindicato de \\
construcción civil
\end{tabular}} & \multirow{5}{*}{$\begin{array}{l}\text { Coordinación } \\
\text { no adecuada } \\
\text { con sindicato }\end{array}$} & \multirow{5}{*}{$\begin{array}{l}\text { Reclamo de } \\
\text { sindicato de } \\
\text { construcción } \\
\text { civil frente } \\
\text { a obra para } \\
\text { incluir a su } \\
\text { personal }\end{array}$} & \multirow{5}{*}{$\begin{array}{l}\text { Construcción de } \\
\text { Tuberías de Conexión } \\
\text { Habilitación de } \\
\text { Instalaciones Internas }\end{array}$} & \multirow{5}{*}{0.1} & Alcance & 0.05 & 0.005 & \multirow{5}{*}{ Muy Bajo } \\
\hline & & & & & & Tiempo & 0.10 & 0.010 & \\
\hline & & & & & & Costo & 0.05 & 0.005 & \\
\hline & & & & & & Calidad & 0.05 & 0.005 & \\
\hline & & & & & & \multicolumn{2}{|c|}{$\begin{array}{l}\text { Total Probabilidad } \mathrm{x} \\
\text { Impacto }\end{array}$} & 0.025 & \\
\hline \multirow{5}{*}{ R002 } & \multirow{5}{*}{\begin{tabular}{|l} 
Paralización de \\
obra por vecinos \\
de la zona \\
en proyectos \\
multifamiliares
\end{tabular}} & \multirow{5}{*}{$\begin{array}{l}\text { Coordinación } \\
\text { no adecuada } \\
\text { con juntas } \\
\text { vecinales }\end{array}$} & \multirow{5}{*}{$\begin{array}{l}\text { Reclamo de } \\
\text { vecinos frente } \\
\text { a obra }\end{array}$} & \multirow{5}{*}{$\begin{array}{l}\text { Construcción de } \\
\text { redes matrices } \\
\text { y/o montantes de } \\
\text { instalaciones internas } \\
\text { Habilitación de } \\
\text { instalaciones Internas }\end{array}$} & \multirow{5}{*}{0.3} & Alcance & 0.05 & 0.015 & \multirow{5}{*}{ Bajo } \\
\hline & & & & & & Tiempo & 0.10 & 0.030 & \\
\hline & & & & & & Costo & 0.05 & 0.015 & \\
\hline & & & & & & Calidad & 0.10 & 0.030 & \\
\hline & & & & & & \multicolumn{2}{|c|}{$\begin{array}{l}\text { Total Probabilidad } \mathrm{x} \\
\text { Impacto }\end{array}$} & 0.09 & \\
\hline \multirow{5}{*}{ R003 } & \multirow{5}{*}{$\begin{array}{l}\text { Demora en inicio } \\
\text { de obra por falta } \\
\text { de permisos } \\
\text { municipales. }\end{array}$} & \multirow{5}{*}{$\begin{array}{l}\text { Coordinación } \\
\text { no adecuada } \\
\text { con municipio }\end{array}$} & \multirow{5}{*}{$\begin{array}{l}\text { Rechazo } \\
\text { de permiso } \\
\text { municipal }\end{array}$} & \multirow{5}{*}{$\begin{array}{l}\text { Trámites y permisos. } \\
\text { Construcción de } \\
\text { Tuberías de Conexión. }\end{array}$} & \multirow{5}{*}{0.1} & Alcance & 0.05 & 0.005 & \multirow{5}{*}{ Muy Bajo } \\
\hline & & & & & & Tiempo & 0.10 & 0.010 & \\
\hline & & & & & & Costo & 0.05 & 0.005 & \\
\hline & & & & & & Calidad & 0.05 & 0.005 & \\
\hline & & & & & & \multicolumn{2}{|c|}{$\begin{array}{l}\text { Total Probabilidad x } \\
\text { Impacto }\end{array}$} & 0.025 & \\
\hline \multirow{5}{*}{ R004 } & \multirow{5}{*}{\begin{tabular}{|l|} 
Paralización de \\
construcción y/o \\
gasificación de \\
redes externas \\
en nuevas zonas \\
o extensiones
\end{tabular}} & \multirow{5}{*}{\begin{tabular}{|l} 
Coordinación \\
inadecuada \\
con sindicatos \\
o municipios
\end{tabular}} & \multirow{5}{*}{$\begin{array}{l}\text { Detención de } \\
\text { labores de } \\
\text { contratistas } \\
\text { en obra }\end{array}$} & \multirow{5}{*}{$\begin{array}{l}\text { Construcción de } \\
\text { Tuberías de Conexión. } \\
\text { Construcción de } \\
\text { redes matrices } \\
\text { y/o montantes de } \\
\text { instalaciones internas. } \\
\text { Habilitación de } \\
\text { Instalaciones Internas. }\end{array}$} & \multirow{5}{*}{0.3} & Alcance & 0.40 & 0.120 & \multirow{5}{*}{ Alto } \\
\hline & & & & & & Tiempo & 0.80 & 0.240 & \\
\hline & & & & & & Costo & 0.40 & 0.120 & \\
\hline & & & & & & Calidad & 0.05 & 0.015 & \\
\hline & & & & & & \multicolumn{2}{|c|}{$\begin{array}{l}\text { Total Probabilidad x } \\
\text { Impacto }\end{array}$} & 0.495 & \\
\hline
\end{tabular}




\begin{tabular}{|c|c|c|c|c|c|c|c|c|c|}
\hline \multirow{5}{*}{ R005 } & \multirow{5}{*}{$\begin{array}{l}\text { Paralización o } \\
\text { demora de obra } \\
\text { por no disponer } \\
\text { de materiales }\end{array}$} & \multirow{5}{*}{$\begin{array}{l}\text { Mala } \\
\text { coordinación } \\
\text { con } \\
\text { proveedores }\end{array}$} & \multirow{5}{*}{$\begin{array}{l}\text { Falta de } \\
\text { materiales } \\
\text { para iniciar } \\
\text { o continuar } \\
\text { trabajos }\end{array}$} & \multirow{5}{*}{$\begin{array}{l}\text { Construcción de } \\
\text { Tuberías de Conexión. } \\
\text { Construcción de } \\
\text { redes matrices } \\
\text { y/o montantes de } \\
\text { instalaciones internas. } \\
\text { Habilitación de } \\
\text { Instalaciones Internas. }\end{array}$} & \multirow{5}{*}{0.3} & Alcance & 0.80 & 0.240 & \multirow{5}{*}{ Alto } \\
\hline & & & & & & Tiempo & 0.40 & 0.120 & \\
\hline & & & & & & Costo & 0.05 & 0.015 & \\
\hline & & & & & & Calidad & 0.10 & 0.030 & \\
\hline & & & & & & \multicolumn{2}{|c|}{$\begin{array}{l}\text { Total Probabilidad } x \\
\text { Impacto }\end{array}$} & 0.405 & \\
\hline \multirow{5}{*}{ R006 } & \multirow{5}{*}{$\begin{array}{l}\text { Incidentes con } \\
\text { trabajos en } \\
\text { caliente }\end{array}$} & \multirow{5}{*}{$\begin{array}{l}\text { Mala } \\
\text { operación } \\
\text { / Falla en } \\
\text { aplicación } \\
\text { de procedi- } \\
\text { mientos }\end{array}$} & \multirow{5}{*}{$\begin{array}{l}\text { Amago de } \\
\text { incendio / } \\
\text { Accidentes } \\
\text { personales } \\
\text { incapacitantes }\end{array}$} & \multirow{5}{*}{$\begin{array}{l}\text { Construcción de } \\
\text { Tuberías de Conexión. } \\
\text { Construcción de } \\
\text { redes matrices } \\
\text { y/o montantes de } \\
\text { instalaciones internas. } \\
\text { Habilitación de } \\
\text { Instalaciones Internas. }\end{array}$} & \multirow{5}{*}{0.5} & Alcance & 0.05 & 0.025 & \multirow{5}{*}{$\begin{array}{l}\text { Mode- } \\
\text { rado }\end{array}$} \\
\hline & & & & & & Tiempo & 0.10 & 0.050 & \\
\hline & & & & & & Costo & 0.20 & 0.100 & \\
\hline & & & & & & Calidad & 0.10 & 0.050 & \\
\hline & & & & & & \multicolumn{2}{|c|}{$\begin{array}{l}\text { Total Probabilidad } x \\
\text { Impacto }\end{array}$} & 0.225 & \\
\hline \multirow{5}{*}{ R007 } & \multirow{5}{*}{$\begin{array}{l}\text { Incumplimiento } \\
\text { del servicio } \\
\text { designado por } \\
\text { contrato marco }\end{array}$} & \multirow{5}{*}{$\begin{array}{l}\text { Falta de } \\
\text { personal } \\
\text { capacitado } \\
\text { para labor } \\
\text { determinada }\end{array}$} & \multirow{5}{*}{$\begin{array}{l}\text { Detección de } \\
\text { incumplimien- } \\
\text { tos en la } \\
\text { calidad, } \\
\text { seguridad, } \\
\text { plazo de } \\
\text { entrega del } \\
\text { servicio }\end{array}$} & \multirow{5}{*}{$\begin{array}{l}\text { Construcción de } \\
\text { Tuberías de Conexión. } \\
\text { Construcción de } \\
\text { redes matrices } \\
\text { y/o montantes de } \\
\text { instalaciones internas. } \\
\text { Habilitación de } \\
\text { Instalaciones Internas. }\end{array}$} & \multirow{5}{*}{0.5} & Alcance & 0.05 & 0.025 & \multirow{5}{*}{ Alto } \\
\hline & & & & & & Tiempo & 0.20 & 0.100 & \\
\hline & & & & & & Costo & 0.20 & 0.100 & \\
\hline & & & & & & Calidad & 0.40 & 0.200 & \\
\hline & & & & & & \multicolumn{2}{|c|}{$\begin{array}{l}\text { Total Probabilidad } x \\
\text { Impacto }\end{array}$} & 0.425 & \\
\hline \multirow{5}{*}{ R008 } & \multirow{5}{*}{$\begin{array}{l}\text { Retiro de } \\
\text { contratista }\end{array}$} & \multirow{5}{*}{$\begin{array}{l}\text { Mala } \\
\text { evaluación } \\
\text { de contratista } \\
\text { / Cambio } \\
\text { de rubro } \\
\text { de trabajo / } \\
\text { Quiebra }\end{array}$} & \multirow{5}{*}{$\begin{array}{l}\text { Acuerdo para } \\
\text { liquidación de } \\
\text { contrato }\end{array}$} & \multirow{5}{*}{$\begin{array}{l}\text { Construcción de } \\
\text { Tuberías de Conexión. } \\
\text { Construcción de } \\
\text { redes matrices } \\
\text { y/o montantes de } \\
\text { instalaciones internas. } \\
\text { Habilitación de } \\
\text { Instalaciones Internas. }\end{array}$} & & Alcance & 0.40 & 0.12 & \\
\hline & & & & & & Tiempo & 0.40 & 0.12 & \\
\hline & & & & & & Costo & 0.10 & 0.03 & \\
\hline & & & & & 0.3 & Calidad & 0.10 & 0.03 & Mode- \\
\hline & & & & & & $\begin{array}{l}\text { Total Probab } \\
\text { Impacto }\end{array}$ & ilidad $x$ & 0.30 & rado \\
\hline & & & & & & Alcance & 0.40 & 0.120 & \\
\hline & & & & $\begin{array}{l}\text { Construccion de } \\
\text { Tuberías de Conexión. }\end{array}$ & & Tiempo & 0.40 & 0.120 & \\
\hline & Paralización de & $\mid \begin{array}{l}\text { Retrasos en } \\
\text { verificación de }\end{array}$ & Falta o & Construcción de & & Costo & 0.05 & 0.015 & \\
\hline R009 & obras por falta & información & retrasos & redes matrices & 0.3 & Calidad & 0.05 & 0.015 & Mode- \\
\hline & económica & $\begin{array}{l}\text { de pagos en } \\
\text { SAP }\end{array}$ & contratistas & $\begin{array}{l}\text { instalaciones internas. } \\
\text { Habilitación de } \\
\text { Instalaciones Internas. }\end{array}$ & & $\begin{array}{l}\text { Total Probak } \\
\text { Impacto }\end{array}$ & ilidad $\mathrm{x}$ & 0.27 & \\
\hline & & & & & & Alcance & 0.40 & 0.120 & \\
\hline & & & & $\begin{array}{l}\text { Construccion de } \\
\text { Tuberías de Conexión. }\end{array}$ & & Tiempo & 0.40 & 0.120 & \\
\hline & Paralización & Retrasos & & Construcción de & & Costo & 0.05 & 0.015 & \\
\hline R010 & de obras & en pagos a & $\begin{array}{l}\text { Huelga de } \\
\text { instaladores y }\end{array}$ & redes matrices & 0.3 & Calidad & 0.05 & 0.015 & Mode- \\
\hline & contratista & Descuentos & habilitadores & $\begin{array}{l}\text { instalaciones internas. } \\
\text { Habilitación de } \\
\text { Instalaciones Internas }\end{array}$ & & $\begin{array}{l}\text { Total Probak } \\
\text { Impacto }\end{array}$ & ilidad $x$ & 0.27 & \\
\hline
\end{tabular}

Fuente: Elaboración propia. 


\section{CONCLUSIONES}

Conforme al diagnóstico de riesgos se aprecia que la mayoría de los riesgos califica como moderado y se deben establecer medidas de control para la reducción de riesgos.

La determinación de riesgos en una segunda intervención permitirá generar compromisos entre el personal que se encuentra dentro del área de riesgos moderados.

Dentro de las medidas de prevención que se van a implementar para mitigar y/o eliminar los riesgos operacionales, están: chequear periódica mente el funcionamiento de todos los equipos de la red principal y estaciones de regulación y medición.

Las causas de riesgos operacionales se deben minimizar al $100 \%$ para que estas no sean de alto impacto a la industria del gas natural.

Se puede concluir que este trabajo sobre evaluación de riesgos físicos e implementación de medidas de protección frente a riesgos patrimoniales, además de los beneficios económicos en la reducción de pérdidas económicas, trae consigo un mejoramiento en el capital intelectual como es la cultura organizacional, imagen corporativa y protección del recurso humano.

\section{RECOMENDACIONES}

En el plan de gestión de riesgos de distribución de gas natural deben considerarse las funciones $y$ responsabilidades de los integrantes del mismo, para cada una de las actividades tales como activación de alarma, búsqueda, rescate y evacuación, control de incendios y atención de primeros auxilios.

La propuesta para mitigar los riesgos evaluados en la matriz de protección debe ser implementada, a través del uso de la tecnología y de la capacitación del recurso humano. Debe realizarse una evaluación posterior a la implementación del proyecto para determinar en qué medida se ha reducido los riesgos y su justificación según el análisis costo beneficio.

Registrar los riesgos y causas que los originaron, así como las medidas para disminuirlos.

Además se recomienda una evaluación de riesgos contra incendio, que incluya las medidas de protección de las personas, factores de peligro esenciales y la definición de las medidas necesarias para cubrir el riesgo. Esta evaluación técnica permitirá abordar y justificar la implementación de un plan contra incendios, que fue identificada en la evaluación de riesgos físicos.

Mejorar día a día los procesos de capacitación del personal.

Actualizar todos y cada uno de los manuales de procedimientos relacionados a cada área en cuestión.

Mantener al margen la dosificación del equipo y herramienta necesaria que se requiere en las áreas técnicas y de operación.

\section{REFERENCIAS BIBLIOGRÁFICAS}

[1] ABB (2011). Petroleo y gas. Petroleo y gas, 4-5.

[2] Chambergo, O. (2009). Aspectos Esenciales del Gas Natural: Un Enfoque que Contribuye a Promover su Desarrollo. La Revista del Gas Natural, 18-29.

[3] Eyssautier, M. (2006). Metodología de la investigación. Mexico: Cengage Learning Editores.

[4] Fernández, R. (2009). Gas, Petróleo e Imperialismo en Bolivia. La Paz: Plural Editores.

[5] Inkpen, Moffett. (2011). The Global Oil \& Gas Industry: Management, Strategy \& Finance. USA: Pennwell.

[6] Lopez, M. (2010). Evaluación Técnico Económica de las Alternativas Tecnológicas de Transporte de Gas Natural . La Revista del Gas Natural, 18-26.

[7] Parra, E. (2003). Petróleo y gas natural. Madrid: Ediciones Akal S.A.

[8] PMI (2008). Guía de los fundamentos para la dirección de proyectos (Guía del PMBOK). Estados Unidos de América: Project Management Institute, Inc.

[9] Raymond, Leffler (2006). Oil \& Gas Production in Nontechnical Language. USA: Pennwell. 